Appl. Set-Valued Anal. Optim. 2 (2020), No. 3, pp. 285-293

Available online at http://asvao.biemdas.com

https://doi.org/10.23952/asvao.2.2020.3.03

\title{
ITERATIVE ALGORITHMS FOR A MULTIPLE-SETS SPLIT FEASIBILITY PROBLEM IN BANACH SPACES
}

\author{
FENGHUI WANG \\ Department of Mathematics, Luoyang Normal University, Luoyang 471934, China
}

\begin{abstract}
In this paper, we consider a multiple-sets split feasibility problem in Banach spaces. By converting it to a fixed point problem, we propose two new iterative algorithms for solving the problem considered. Under some mild assumptions, we prove that the proposed algorithms are strongly convergent provided that the involved spaces are smooth and uniformly convex.
\end{abstract}

Keywords. Split feasibility problem; Bregman projection; Metric projection; Duality mapping.

\section{INTRODUCTION}

The split feasibility problem (SFP) is formulated as finding a point $x^{*} \in \mathbb{R}^{n}$ satisfying the property:

$$
x^{*} \in C \text { and } A x^{*} \in Q,
$$

where $C$ and $Q$ are nonempty closed convex subset of $\mathbb{R}^{n}$ and $\mathbb{R}^{m}$, respectively, and $A$ is an $m \times n$ matrix (i.e., a linear operator from $\mathbb{R}^{n}$ into $\mathbb{R}^{m}$ ). The SFP was first introduced by Censor and Elfving [7] and has been proved very useful in dealing with problems arising from various applied disciplines; see e.g [5, 6, 9, 10, 22].

There are various entensions of the SFP recently. In this paper, we mainly focus on the multiple-sets split feasibility problem (MSSFP) in Banach spaces: For $m \in \mathbb{N}$ and for two Banach spaces $X$ and $Y$, find $x^{*} \in \bigcap_{i=1}^{m} C_{i}$ such that

$$
A_{i} x^{*} \in Q_{i} \text {, for each } i=1,2, \cdots, m,
$$

where $C_{i}$ and $Q_{i}$ are nonempty closed convex subset of $X$ and $Y$, respectively, and $A_{i}$ is a linear operator from $X$ into $Y$.

In the setting of Hilbert spaces, various algorithms were invented to solve the SFP (1.1) (see $[4,9,10,11,16,19,20,21]$ and reference therein). In 2002, Byrne introduced his $C Q$ algorithm:

$$
x_{n+1}=P_{C}\left[x_{n}-r A^{*}\left(I-P_{Q}\right) A x_{n}\right],
$$

where $0<r_{n}<\frac{2}{\|A\|^{2}}, A^{*}$ is the transpose of $A, I$ is the identity operator, $P_{C}$ denote the metric projection onto $C$, and $P_{Q}$ denotes the metric projection onto $Q$. By using the Polyak's gradient

E-mail address:wfenghui@lynu.edu.cn.

Received July 19, 2020; Accepted December 2, 2020.

(C)2020 Applied Set-Valued Analysis and Optimization 
method, Wang [17] recently proposed another iterative algorithm:

$$
x_{n+1}=x_{n}-r_{n}\left[\left(I-P_{C}\right) x_{n}+A^{*}\left(I-P_{Q}\right) A x_{n}\right],
$$

where $0<r_{n}<\frac{2}{1+\|A\|^{2}}$ and $I$ denotes the identity operaotr.

In the setting of Banach spaces, Schöpfer, Schuster and Louis [12] considered the SFP in Banach spaces and they extended the CQ method as:

$$
x_{n+1}=\Pi_{C} J_{X^{*}}\left[J_{X} x_{n}-r A^{*} J_{Y}\left(I-P_{Q}\right) A x_{n}\right],
$$

where $J_{X^{*}}, J_{X}, J_{Y}$ are the duality mappings, $\Pi_{C}$ denotes the Bregman projection and $P_{Q}$ denotes the metric projection. Then the weak convergence of (1.5) is guaranteed if $X$ is $p$-uniformly convex, uniformly smooth, and $J_{X}$ is sequentially weak-to-weak continuous; see [12] for more details.

Recently, Takahashi [14] suggested a novel way for the SFP:

$$
\left\{\begin{array}{l}
z_{n}=x_{n}-r J_{X^{*}} A^{*} J_{Y}\left(I-P_{Q}\right) A x_{n} \\
C_{n}=\left\{z \in C:\left\langle z_{n}-z, J_{X}\left(x_{n}-z_{n}\right)\right\rangle \geq 0\right\} \\
Q_{n}=\left\{z \in C:\left\langle x_{n}-z, J_{X}\left(x_{0}-x_{n}\right)\right\rangle \geq 0\right\} \\
x_{n+1}=P_{C_{n} \cap Q_{n}}\left(x_{0}\right) .
\end{array}\right.
$$

Instead of the weak convergence, Takahashi proved the strong convergence of method (1.6) under the assumption that $X$ is uniformly convex and smooth, which is clearly weaker than that used in [12].

Motivated by this method, Wang [18] recently introduced another new method as

$$
\left\{\begin{array}{l}
z_{n}=x_{n}-r J_{X^{*}}\left[J_{X}\left(I-P_{C}\right) x_{n}+A^{*} J_{Y}\left(I-P_{Q}\right) A x_{n}\right], \\
C_{n}=\left\{z \in X:\left\langle z_{n}-z, J_{X}\left(x_{n}-z_{n}\right)\right\rangle \geq 0\right\}, \\
Q_{n}=\left\{z \in X:\left\langle x_{n}-z, J_{X}\left(x_{0}-x_{n}\right)\right\rangle \geq 0\right\} \\
x_{n+1}=P_{C_{n} \cap Q_{n}}\left(x_{0}\right) .
\end{array}\right.
$$

It is clear that $C_{n}$ and $Q_{n}$ constructed in (1.7) are halfspaces. Since the projection onto the intersection of two half spaces has closed-form soulution, it is easy to implement the method (1.7). For some related works on this topic, we refer to $[11,15,16]$.

The aim in this paper is to continue the above works by constructing new iterative methods for solving the MSSFP. By converting it to an equivalent fixed point problem, we propose two iterative algorithms and prove their strong convergence to a solution of the MSSFP. It is also worth noting that the parameter in one algorithm is chosen in a way that no priori knowledge of the operator norms are required.

\section{PRELIMINARIES}

NOTATION: " $\rightarrow$ " denotes strong convergence, " - " denoes weak convergence and $\omega_{w}\left\{x_{n}\right\}$ denotes the set of weak cluster points of a sequence $\left\{x_{n}\right\}$. Let $S_{X}=\{x \in X:\|x\|=1\}$ and $B_{X}=$ $\{x \in X:\|x\| \leq 1\}$, respectively, be the unit sphere and unit ball of $X$. Fix $(T)=\{x \in X: T x=x\}$ denotes the fixed-point set of $T$.

Definition 2.1. Let $X$ be a Banach space.

(1) The modulus of convexity $\delta_{X}(\varepsilon):[0,2] \rightarrow[0,1]$ is defined as

$$
\delta_{X}(\varepsilon)=\inf \left\{1-\frac{\|x+y\|}{2}: x, y \in B_{X},\|x-y\| \geq \varepsilon\right\} .
$$


(2) The modulus of smoothness $\rho_{X}(t):[0, \infty) \rightarrow[0, \infty)$ is defined by

$$
\rho_{X}(t)=\sup \left\{\frac{\|x+t y\|+\|x-t y\|}{2}-1: x, y \in S_{X}\right\} .
$$

(3) The duality mapping $J_{X}: X \rightarrow 2^{X^{*}}$ is defined by

$$
J_{X} x=\left\{x^{*} \in X^{*}:\left\langle x, x^{*}\right\rangle=\|x\|^{2}=\left\|x^{*}\right\|^{2}\right\} .
$$

Definition 2.2. Let $X$ be a Banach space.

(1) $X$ is called strictly convex if $\delta_{X}(2)=1$.

(2) $X$ is called uniformly convex if $\delta_{X}(\varepsilon)>0$ for any $\varepsilon \in(0,2]$.

(3) $X$ is called $p$-uniformly convex if there exist $p \geq 2$ and a constant $c>0$ such that $\delta_{X}(\varepsilon) \geq$ $c \varepsilon^{p}, \forall \varepsilon \in(0,2]$.

Definition 2.3. Let $X$ be a Banach space.

(1) $X$ is called smooth if $\lim _{t \rightarrow 0} \frac{\|x+t y\|-\|x\|}{t}$ exists for each $x, y \in S_{X}$.

(2) $X$ is called uniformly smooth if $\lim _{t \rightarrow 0} \frac{\rho_{X}(t)}{t}=0$.

Lemma 2.1. $[8,13]$ Let $X$ be a uniformly convex Banach space. Then $X^{*}$ is uniformly smooth and $X$ is strictly convex and reflexive.

Lemma 2.2. $[8,13]$ Let $X$ be a uniformly convex Banach space. Let $\left\{x_{n}\right\}$ be a sequence in $X$. If $x_{n} \rightarrow x$ and $\left\|x_{n}\right\| \rightarrow\|x\|$, then the sequence $\left\{x_{n}\right\}$ converges strongly to $x$.

Lemma 2.3. $[8,13]$ If $X$ is reflexive, smooth and strictly convex, then the duality mapping $J_{X}$ is one-to-one, single-valued and $J_{X}^{-1}=J_{X^{*}}$, where $J_{X^{*}}$ is the duality mapping of $X^{*}$.

Definition 2.4. Let $C$ be a nonempty closed convex subset of $X$. The metric projection $P_{C}: X \rightarrow$ $C$ is defined as

$$
P_{C} x:=\arg \min _{y \in C}\|x-y\|, \quad x \in X .
$$

Lemma 2.4. $[11,12]$ Let $\left\{x_{n}\right\}$ be a sequence in $X$, and $C \subseteq X$ a nonempty closed convex subset. Then, for $x \in X$,

(1) $\left\langle J_{X}\left(x-P_{C} x\right), z-P_{C} x\right\rangle \leq 0, \forall z \in C$;

(2) $\left\|x-P_{C} x\right\|^{2} \leq\left\langle J_{X}\left(x-P_{C} x\right), x-z\right\rangle, \forall z \in C$;

(3) if $x_{n} \rightarrow x$ and $\left\|x_{n}-P_{C} x_{n}\right\| \rightarrow 0$, then $x \in C$.

The Bregman distance with respect to $\|\cdot\|$ is given by

$$
\Delta(x, y)=\frac{1}{2}\|x\|^{2}-\left\langle J_{X} x, y\right\rangle+\frac{1}{2}\|y\|^{2} .
$$

This notion goes back to Bregman [3] and now is successfully used in various optimization problems in Banach spaces; see, e.g., [1,2]. In general, the Bregman distance is not a metric due to the absence of symmetry, but it has some distance-like properties.

Definition 2.5. Let $C$ be a nonempty closed convex subset of $X$. The Bregman projection $\Pi_{C}$ : $X \rightarrow C$ is defined as

$$
\Pi_{C} x=\arg \min _{y \in C} \triangle(x, y), \quad x \in X
$$


In Hilbert spaces, the metric and Bregman projections are the same, but in general they are completely different. More importantly, the metric projection can not share the decent property:

$$
\triangle\left(\Pi_{C} x, z\right) \leq \triangle(x, z)-\triangle\left(x, \Pi_{C} x\right), \forall z \in C,
$$

as the Bregman projection in Banach spaces.

\section{REFORMULATION FOR MSSFP}

To construct our algorithm, we first convert the MSSFP to an equivalent fixed point problem. As a matter of fact, for each $i \in\{1,2, \cdots, m\}$, let us define

$$
W:=I-r J_{X}^{*}\left[\sum_{i=1}^{m}\left(J_{X} U_{i}+A_{i}^{*} J_{Y} T_{i} A_{i}\right)\right],
$$

where $r>0, U_{i}=I-P_{C_{i}}$ and $T_{i}=I-P_{Q_{i}}$.

Lemma 3.1. Assume that both $X$ and $Y$ are smooth, reflexive, and strictly convex. If the MSSFP is consistent, that is, its solution set $\mathscr{S}$ is nonempty, then $\mathscr{S}=\operatorname{Fix}(W)$.

Proof. By our assumption, both $J_{X}$ and $J_{Y}$ are one-to-one and single-valued. It is readily seen that $\mathscr{S} \subseteq \operatorname{Fix}(W)$. To see the converse, let $x^{*} \in \operatorname{Fix}(W)$ and choose any element $z$ is in $\mathscr{S}$. By Lemma 2.4, for each $i \in\{1,2, \cdots, m\}$, we have

$$
\begin{aligned}
\left\|U_{i} x^{*}\right\|^{2} & \leq\left\langle J_{X} U_{i} x^{*}, x^{*}-z\right\rangle, \\
\left\|T_{i} A_{i} x^{*}\right\|^{2} & \leq\left\langle A_{i}^{*} J_{Y} T_{i} A_{i} x^{*}, x^{*}-z\right\rangle .
\end{aligned}
$$

Combining these inequalities, we have

$$
\begin{aligned}
& r \sum_{i=1}^{m}\left(\left\|U_{i} x^{*}\right\|^{2}+\left\|T_{i} A_{i} x^{*}\right\|^{2}\right) \\
& \leq r\left\langle\sum_{i=1}^{m}\left(J_{X} U_{i} x^{*}+A_{i}^{*} J_{Y} T_{i} A_{i} x^{*}\right), x^{*}-z\right\rangle \\
& =\left\langle J_{X}\left(x^{*}-W x^{*}\right), x^{*}-z\right\rangle=0,
\end{aligned}
$$

which yields $x^{*} \in \mathscr{S}$. Thus the desired result follows.

\section{Algorithms FOR MSSFP}

As an application of Lemma 3.1, we propose a new algorithm for solving the MSSFP, which is indeed an extension of algorithm (1.7).

Algorithm 4.1. Choose an arbitrary initial guess $x_{0} \in X$. Given $x_{n}$, update $x_{n+1}$ by the iteration formula:

$$
\left\{\begin{array}{l}
C_{n}=\left\{z \in X:\left\langle W x_{n}-z, J_{X}\left(x_{n}-W x_{n}\right)\right\rangle \geq 0\right\} \\
Q_{n}=\left\{z \in X:\left\langle x_{n}-z, J_{X}\left(x_{0}-x_{n}\right)\right\rangle \geq 0\right\} \\
x_{n+1}=P_{C_{n} \cap Q_{n}}\left(x_{0}\right),
\end{array}\right.
$$

where $W$ is defined as in (3.1) and the parameter $r_{n}$ is chosen so that

$$
0<r \leq r_{n} \leq \frac{1}{1+\max _{1 \leq i \leq m}\left\|A_{i}\right\|^{2}} \text {. }
$$


Lemma 4.1. Assume that both $X$ and $Y$ are smooth, reflexive, and strictly convex Banach spaces. If the MSSFP is consistent, then, for each each $n \in \mathbb{N}$, the set $C_{n} \cap Q_{n}$ is nonempty, closed and convex. Moreover the sequence $\left\{x_{n}\right\}$ generated by Algorithm 4.1 is well defined.

Proof. For any $z \in \mathscr{S}$, we have

$$
\begin{aligned}
& \left\langle x_{n}-z, J_{X}\left(x_{n}-W x_{n}\right)\right\rangle \\
& =r_{n}\left\langle x_{n}-z, \sum_{i=1}^{m}\left(J_{X} U_{i} x_{n}+A_{i}^{*}\left(J_{Y}\left(T_{i}\left(A_{i} x_{n}\right)\right)\right)\right)\right\rangle \\
& \left.=r_{n} \sum_{i=1}^{m}\left(\left\langle x_{n}-z, J_{X} U_{i} x_{n}\right\rangle+\left\langle A_{i} x_{n}-A_{i} z, J_{Y}\left(T_{i}\left(A_{i} x_{n}\right)\right)\right)\right\rangle\right) .
\end{aligned}
$$

It then follows from Lemma 2.4 that

$$
\left\langle x_{n}-z, J_{X}\left(x_{n}-W x_{n}\right)\right\rangle \geq r_{n} \sum_{i=1}^{m}\left(\left\|U_{i} x_{n}\right\|^{2}+\left\|T_{i}\left(A_{i} x_{n}\right)\right\|^{2}\right),
$$

which implies

$$
\begin{aligned}
& \left\langle W x_{n}-z, J_{X}\left(x_{n}-W x_{n}\right)\right\rangle \\
& =\left\langle W x_{n}-x_{n}, J_{X}\left(x_{n}-W x_{n}\right)\right\rangle+\left\langle x_{n}-z, J_{X}\left(x_{n}-W x_{n}\right)\right\rangle \\
& =-\left\|W x_{n}-x_{n}\right\|^{2}+\left\langle x_{n}-z, J_{X}\left(x_{n}-W x_{n}\right)\right\rangle \\
& \geq r_{n} \sum_{i=1}^{m}\left(\left\|U_{i} x_{n}\right\|^{2}+\left\|T_{i}\left(A_{i} x_{n}\right)\right\|^{2}\right)-\left\|x_{n}-W x_{n}\right\|^{2} .
\end{aligned}
$$

On the other hand, by Young's inequality,

$$
\begin{aligned}
\left\|x_{n}-W x_{n}\right\|^{2} & =r_{n}^{2}\left\|\sum_{i=1}^{m}\left(J_{X} U_{i} x_{n}+A_{i}^{*}\left(J_{Y}\left(T_{i}\left(A_{i} x_{n}\right)\right)\right)\right)\right\|^{2} \\
& \leq r_{n}^{2}\left(\sum_{i=1}^{m}\left(\left\|U_{i} x_{n}\right\|+\left\|A_{i}\right\|\left\|T_{i} A_{i} x_{n}\right\|\right)\right)^{2} \\
& \leq r_{n}^{2} \sum_{i=1}^{m}\left(1+\left\|A_{i}\right\|^{2}\right)\left(\left\|U_{i} x_{n}\right\|^{2}+\left\|T_{i}\left(A_{i} x_{n}\right)\right\|^{2}\right) \\
& \leq r_{n}^{2}\left(1+\max _{1 \leq i \leq m}\left\|A_{i}\right\|^{2}\right) \sum_{i=1}^{m}\left(\left\|U_{i} x_{n}\right\|^{2}+\left\|T_{i}\left(A_{i} x_{n}\right)\right\|^{2}\right) .
\end{aligned}
$$

Substituting this into (4.3), we have

$$
\begin{aligned}
& \left\langle W x_{n}-z, J_{X}\left(x_{n}-W x_{n}\right)\right\rangle \\
& \geq r_{n}\left(1-r_{n}\left(1+\max _{1 \leq i \leq m}\left\|A_{i}\right\|^{2}\right)\right) \sum_{i=1}^{m}\left(\left\|U_{i} x_{n}\right\|^{2}+\left\|T_{i}\left(A_{i} x_{n}\right)\right\|^{2}\right) .
\end{aligned}
$$

By condition (4.1), we have $\left\langle W x_{n}-z, J_{X}\left(x_{n}-W x_{n}\right)\right\rangle \geq 0$. Hence, we deduce that $z \in C_{n}$. Since $z$ is chosen in $\mathscr{S}$ arbitrarily, we conclude that $\mathscr{S} \subseteq C_{n}$ for all $n \in \mathbb{N}$.

We next show $\mathscr{S} \subseteq Q_{n}$ by induction. It is easy to check that $\mathscr{S} \subseteq Q_{0}$. Now assume that $\mathscr{S} \subseteq Q_{k}$ for some $k \geq 1$, which implies $\mathscr{S} \subseteq C_{k} \cap Q_{k}$. Since $x_{k+1}$ lies in $C_{k} \cap Q_{k}$, this gives

$$
\left\langle x_{k+1}-z, J_{X}\left(x_{0}-x_{k+1}\right)\right\rangle \geq 0, \forall z \in C_{k} \cap Q_{k} \text {. }
$$


In particular, we have

$$
\left\langle x_{k+1}-z, J_{X}\left(x_{0}-x_{k+1}\right)\right\rangle \geq 0, \forall z \in \mathscr{S},
$$

which implies $\mathscr{S} \subseteq Q_{k+1}$. Hence, $\mathscr{S} \subseteq Q_{n}$ for all $n \in \mathbb{N}$.

Altogether, $\mathscr{S} \subseteq C_{n} \cap Q_{n}$ for all $n \in \mathbb{N}$. This implies that the set $C_{n} \cap Q_{n}$ is nonempty, closed and convex, and therefore iterative sequence $\left\{x_{n}\right\}$ is well defined. Thus, the proof is complete.

Now let us state the convergence of $\left\{x_{n}\right\}$ generated by Algorithm 4.1.

Theorem 4.1. Assume that $X$ is a smooth and uniformly convex Banach space, and $Y$ is a smooth, reflexive, and strictly convex Banach space. If the MSSFP is consistent, then the sequence $\left\{x_{n}\right\}$ generated by Algorithm 4.1 converges strongly to the solution $P_{\mathscr{S}}\left(x_{0}\right)$.

Proof. We first show that $\lim _{n}\left\|x_{n+1}-x_{n}\right\|=0$. Let $z \in S$. By Lemma 4.1, we have

$$
\left\|x_{1}-x_{n+1}\right\|=\left\|x_{1}-P_{C_{n} \cap Q_{n}} x_{1}\right\| \leq\left\|x_{1}-z\right\| .
$$

Hence $\left\{x_{n}\right\}$ is bounded. On the other hand, using Lemma 4.1, we have

$$
\left\|x_{0}-x_{n}\right\|=\left\|x_{0}-P_{Q_{n}} x_{0}\right\| \leq\left\|x_{0}-\frac{x_{n}+x_{n+1}}{2}\right\|,
$$

due to $\left(x_{n}+x_{n+1}\right) / 2 \in Q_{n}$. Similarly, $\left\|x_{0}-x_{n}\right\| \leq\left\|x_{0}-x_{n+1}\right\|$, which implies that $\left\{\left\|x_{0}-x_{n}\right\|\right\}$ is nondecreasing. This together with its boundness yields that $\lim _{n}\left\|x_{0}-x_{n}\right\|$ exists. Let $M$ be such a limit. It is clear that $M>0$ and $\left\|x_{0}-x_{n}\right\| \leq M$ for all $n \in \mathbb{N}$.

From definition of the modulus of convexity, we have

$$
\begin{aligned}
\delta_{X}\left(\frac{\left\|x_{n}-x_{n+1}\right\|}{M}\right) & =\delta_{X}\left(\left\|\frac{x_{n}-x_{0}}{M}-\frac{x_{n+1}-x_{0}}{M}\right\|\right) \\
& \leq 1-\frac{1}{2}\left\|\frac{x_{n}-x_{0}}{M}+\frac{x_{n+1}-x_{0}}{M}\right\| \\
& =1-\frac{1}{M}\left\|x_{0}-\frac{x_{n}+x_{n+1}}{2}\right\| \\
& \leq 1-\frac{\left\|x_{0}-x_{n}\right\|}{M} \rightarrow 0 .
\end{aligned}
$$

Since $X$ is uniformly convex, we assert that $\lim _{n}\left\|x_{n+1}-x_{n}\right\|=0$.

We next show that $\omega_{w}\left\{x_{n}\right\} \subseteq \mathscr{S}$. It follows from Lemma 4.1 that

$$
\left\|x_{n}-W x_{n}\right\|=\left\|x_{n}-P_{C_{n}} x_{n}\right\| \leq\left\|x_{n}-x_{n+1}\right\| .
$$

It then follows that $\lim _{n}\left\|x_{n}-W x_{n}\right\|=0$, which together with (4.2) and (4.1) yields, for $i \in$ $\{1,2, \cdots, m\}$,

$$
\lim _{n \rightarrow \infty}\left\|x_{n}-P_{C_{i}} x_{n}\right\|=\lim _{n \rightarrow \infty}\left\|A_{i} x_{n}-P_{Q_{i}}\left(A_{i} x_{n}\right)\right\|=0 .
$$

Since $\left\{x_{n}\right\}$ is bounded, there exists a subsequence $\left\{x_{n_{k}}\right\}$ of $\left\{x_{n}\right\}$ converging weakly to $x^{*}$. By Lemma 2.4, $x^{*} \in C_{i}$ for each $i \in\{1,2, \cdots, m\}$. On the other hand, since $A_{i} x_{n_{k}} \rightarrow A_{i} x^{*}$, it follows from (4.4) and Lemma 2.4 that $A_{i} x^{*} \in Q_{i}$ for each $i \in\{1,2, \cdots, m\}$. Altogether, we have $x^{*} \in \mathscr{S}$. 
Fixing $x \in \omega_{w}\left(x_{n}\right)$, we see $x^{*} \in \mathscr{S}$, and there exists a subsequence $\left\{x_{n_{k}}\right\}$ of $\left\{x_{n}\right\}$ converging weakly to $x$. It then follows from (i) that

$$
\begin{aligned}
\left\|x_{0}-P_{\mathscr{S}}\left(x_{0}\right)\right\| & \leq\left\|x_{0}-x\right\| \leq \lim _{k \rightarrow \infty}\left\|x_{0}-x_{n_{k}}\right\| \\
& =\lim _{k \rightarrow \infty}\left\|x_{0}-P_{C_{n_{k}-1} \cap Q_{n_{k}-1}}\left(x_{0}\right)\right\| \\
& \leq\left\|x_{0}-P_{\mathscr{S}}\left(x_{0}\right)\right\|,
\end{aligned}
$$

where the first and the last inequalities follow from the property of metric projections, and the second one from the lower semi-continuity of the norm. Hence, $P_{\mathscr{S}}\left(x_{0}\right)=x$ and

$$
\lim _{k \rightarrow \infty}\left\|x_{0}-x_{n_{k}}\right\|=\left\|x_{0}-P_{\mathscr{S}}\left(x_{0}\right)\right\| .
$$

Since $x$ is chosen arbitrarily, this implies that $\omega_{w}\left(x_{n}\right)$ is exactly single-point set, that is, $\left\{x_{n}\right\}$ converges weakly to $P_{\mathscr{S}}\left(x_{0}\right)$. Note that $x_{0}-x_{n_{k}} \rightarrow x_{0}-P_{\mathscr{S}}\left(x_{0}\right)$. By Lemma $2.3, \lim _{k} x_{n_{k}}=$ $P_{\mathscr{S}}\left(x_{0}\right)$. Since $\left\{x_{n}\right\}$ converges weakly, this yields $\lim _{n} x_{n}=P_{\mathscr{S}}\left(x_{0}\right)$ as desired.

Remark 4.1. In Hilbert spaces, the above algorithm is reduced to:

$$
\left\{\begin{array}{l}
C_{n}=\left\{z \in X:\left\langle W x_{n}-z, x_{n}-W x_{n}\right\rangle \geq 0\right\} \\
Q_{n}=\left\{z \in X:\left\langle x_{n}-z, x_{0}-x_{n}\right\rangle \geq 0\right\} \\
x_{n+1}=P_{C_{n} \cap Q_{n}}\left(x_{0}\right) .
\end{array}\right.
$$

Remark 4.2. It worth noting that $C_{n}$ and $Q_{n}$ in our algorithm are half-spaces, while those in (1.6) are not in general. Our condition that ensures the convergence is weaker than that employed in [12].

As we see from Algorithm 4.1, the choice of $r_{n}$ is related to $\left\|A_{i}\right\|$, thus to implement this algorithm, one has to compute the norm $\left\|A_{i}\right\|$, which is generally not easy in practice. In what follows, we introduce another choice of $r_{n}$, which ultimately has no relation with $\left\|A_{i}\right\|$.

Algorithm 4.2. Choose an arbitrary initial guess $x_{0} \in X$. Given $x_{n}$, if

$$
\left\|\sum_{i=1}^{m}\left(J_{X}\left(U_{i} x_{n}\right)+A_{i}^{*}\left(J_{Y} T_{i}\left(A_{i} x_{n}\right)\right)\right)\right\|=0,
$$

then $x_{n}$ is a solution; otherwise, update $x_{n+1}$ by the iteration formula:

$$
\left\{\begin{array}{l}
C_{n}=\left\{z \in X:\left\langle W x_{n}-z, J_{X}\left(x_{n}-W x_{n}\right)\right\rangle \geq 0\right\} \\
Q_{n}=\left\{z \in X:\left\langle x_{n}-z, J_{X}\left(x_{0}-x_{n}\right)\right\rangle \geq 0\right\} \\
x_{n+1}=P_{C_{n} \cap Q_{n}}\left(x_{0}\right)
\end{array}\right.
$$

where $W$ is defined as in (3.1) and the parameter $r_{n}$ is chosen as

$$
r_{n}=\frac{\sum_{i=1}^{m}\left(\left\|U_{i} x_{n}\right\|^{2}+\left\|T_{i}\left(A_{i} x_{n}\right)\right\|^{2}\right)}{\left\|\sum_{i=1}^{m}\left(J_{X}\left(U_{i} x_{n}\right)+A_{i}^{*}\left(J_{Y} T_{i}\left(A_{i} x_{n}\right)\right)\right)\right\|^{2}} .
$$

Lemma 4.2. Assume that both $X$ and $Y$ are smooth, reflexive, and strictly convex Banach spaces. If the MSSFP is consistent, then, for each $n \in \mathbb{N}$, the set $C_{n} \cap Q_{n}$ is nonempty, closed and convex. Moreover, the sequence $\left\{x_{n}\right\}$ generated by Algorithm 4.2 is well defined. 
Proof. From the proof of Lemma 4.1, it suffices to show that $\left\langle W x_{n}-z, J_{X}\left(x_{n}-W x_{n}\right)\right\rangle \geq 0$ for each $n \in \mathbb{N}$. To see this, let $z \in \mathscr{S}$. It then follows from (4.3) that

$$
\begin{aligned}
& \left\langle W x_{n}-z, J_{X}\left(x_{n}-W x_{n}\right)\right\rangle \\
& \geq r_{n}\left(\sum_{i=1}^{m}\left(\left\|U_{i} x_{n}\right\|^{2}+\left\|T_{i}\left(A_{i} x_{n}\right)\right\|^{2}\right)-\left\|x_{n}-W x_{n}\right\|^{2}\right. \\
& =r_{n}\left(\sum_{i=1}^{m}\left(\left\|U_{i} x_{n}\right\|^{2}+\left\|T_{i}\left(A_{i} x_{n}\right)\right\|^{2}\right)-r_{n}^{2}\left\|\sum_{i=1}^{m}\left(J_{X}\left(U_{i} x_{n}\right)+A_{i}^{*}\left(J_{Y} T_{i}\left(A_{i} x_{n}\right)\right)\right)\right\|^{2} .\right.
\end{aligned}
$$

By our choice of $r_{n}$, we have the desired inequality immediately.

Now let us state the convergence of $\left\{x_{n}\right\}$ generated by Algorithm 2 .

Theorem 4.2. Assume that $X$ is a smooth and uniformly convex Banach space, and $Y$ is a smooth, reflexive, and strictly convex Banach space. Let $\left\{x_{n}\right\}$ be generated by Algorithm 4.2. If the MSSFP is consistent, then $\left\{x_{n}\right\}$ converges strongly to the solution $P_{\mathscr{S}}\left(x_{0}\right)$.

Proof. From the proof of Theorem 4.1, it suffices to verify that (4.4) still holds true. As a matter of fact, by inequality (4.2), it follows that

$$
\lim _{n \rightarrow \infty} r_{n}\left(\sum_{i=1}^{m}\left(\left\|U_{i} x_{n}\right\|^{2}+\left\|T_{i}\left(A_{i} x_{n}\right)\right\|^{2}\right)=0 .\right.
$$

On the other hand, we see that

$$
\begin{aligned}
r_{n} & =\frac{\sum_{i=1}^{m}\left(\left\|U_{i} x_{n}\right\|^{2}+\left\|T_{i}\left(A_{i} x_{n}\right)\right\|\right)^{2}}{\left\|\sum_{i=1}^{m}\left(J_{X}\left(U_{i} x_{n}\right)+A_{i}^{*}\left(J_{Y} T_{i}\left(A_{i} x_{n}\right)\right)\right)\right\|^{2}} \\
& \geq \frac{\sum_{i=1}^{m}\left(\left\|U_{i} x_{n}\right\|^{2}+\left\|T_{i}\left(A_{i} x_{n}\right)\right\|\right)^{2}}{\left(\left\|J_{X}\left(x_{n}-P_{C} x_{n}\right)\right\|+\|A\|\left\|J_{Y}\left(A x_{n}-P_{Q_{j}}\left(A x_{n}\right)\right)\right\|\right)^{2}} \\
& \geq \frac{\sum_{i=1}^{m}\left(\left\|U_{i} x_{n}\right\|^{2}+\left\|T_{i}\left(A_{i} x_{n}\right)\right\|\right)^{2}}{\left(1+\max _{1 \leq i \leq m}\left\|A_{i}\right\|^{2}\right)\left(\sum_{i=1}^{m}\left(\left\|U_{i} x_{n}\right\|^{2}+\left\|T_{i}\left(A_{i} x_{n}\right)\right\|^{2}\right)\right.} \\
& \geq \frac{1}{1+\max _{1 \leq i \leq m}\left\|A_{i}\right\|^{2}} \\
& >0 .
\end{aligned}
$$

This together with (4.6) yields (4.4) as desired.

\section{Acknowledgments}

This paper was supported by Key Scientific Research Projects of Universities in Henan Province (No. 19B110010, 20A110029).

\section{REFERENCES}

[1] Y. Alber, D. Butnariu, Convergence of Bregman projection methods for solving consistent convex feasibility problems in reflexive Banach spaces, J. Optim. Theory Appl. 92 (1997), 33-61.

[2] H.H. Bauschke, J.M. Borwein. P.L. Combettes, Bregman monotone optimization algorithms, SIAM J. Control Optim. 42 (2003), 596-636. 
[3] L.M. Bregman, The relaxation method of finding the common point of convex sets and its application to the solution of problems in convex programming, USSR Comput. Math. Math. Phys. 7 (1967), 200-217.

[4] C. Byrne, Iterative oblique projection onto convex sets and the split feasibility problem, Inverse Probl. 18 (2002), 441-453.

[5] C. Byrne, A unified treatment of some iterative algorithms in signal processing and image reconstruction, Inverse Probl. 20 (2004), 103-120.

[6] Y. Censor, T. Bortfeld, B. Martin and A. Trofimov, A unified approach for inversion problems in intensitymodulated radiation therapy, Phys. Med. Biol. 51 (2006), 2353-2365.

[7] Y. Censor, T. Elfving, A multiprojection algorithms using Bregman projection in a product space, Numer. Algo. 8 (1994), 221-239.

[8] I. Cioranescu, Geometry of Banach spaces, duality mappings and nonlinear problems, Kluwer, Dordrecht, 1990.

[9] P.L. Combettes, V.R. Wajs, Signal recovery by proximal forward-backward splitting, Multiscale Model. Simul. 4 (2005), 1168-1200.

[10] G. López, V. Martín, F. Wang, H.K. Xu, Solving the split feasibility problem without prior knowledge of matrix norms, Inverse Probl. 28 (2012), 085004.

[11] F. Schöpfer, Iterative regularization method for the solution of the split feasibility problem in Banach spaces, $\mathrm{PhD}$ thesis, Saarbrücken, 2007.

[12] F. Schöpfer, T. Schuster, A.K. Louis, An iterative regularization method for the solution of the split feasibility problem in Banach spaces, Inverse Probl. 24 (2008), 055008.

[13] W. Takahashi, Nonlinear Functional Analysis, Yokohama Publishers, Yokohama, 2000.

[14] W. Takahashi, The split feasibility problem in Banach spaces, J. Nonlinear Convex Anal. 15 (2014), 13491355.

[15] W. Takahashi, The split feasibility problem and the shrinking projection method in Banach spaces, J. Nonlinear Convex Anal., 16 (2015), 1449-1459.

[16] F. Wang, A new algorithm for solving the multiple-sets split feasibility problem in Banach spaces, Numer. Funct. Anal. Optim. 35 (2014), 99-110.

[17] F. Wang, Polyak's gradient method for split feasibility problem constrained by level sets, Numerical Algo. 77 (2018), 925-938.

[18] F. Wang, Strong convergence of two algorithms for the split feasibility problem in Banach spaces, Optimization, 67 (2018), 1649-1660.

[19] F. Wang, H.K. Xu, Choices of variable steps of the CQ algorithm for the split feasibility problem, Fixed Point Theory, 12 (2011), 489-496.

[20] F. Wang, H.K. Xu, Cyclic algorithms for split feasibility problems in Hilbert spaces, Nonlinear Anal. 74 (2011), 4105-4111.

[21] H.K. Xu, A variable Krasnosel'skii-Mann algorithm and the multiple-set split feasibility problem, Inverse Probl. 22 (2006), 2021-2034.

[22] H. K. Xu, Properties and iterative methods for the Lasso and its variants, Chinese Annals Math. Ser. B 35 (2014), 501-518. 